\title{
Glucose-6-phosphate dehydrogenase plays a crucial role in protection from redox-stress-induced apoptosis
}

\author{
A Fico ${ }^{1}$, F Paglialunga ${ }^{1}$, L Cigliano ${ }^{2}$, P Abrescia ${ }^{2}$, P Verde ${ }^{1}$, \\ G Martini ${ }^{1}$, I laccarino ${ }^{1,3}$ and S Filosa ${ }^{1,3, *}$ \\ 1 Institute of Genetics and Biophysics, 'Adriano Buzzati Traverso', CNR, Via \\ Marconi 10, Napoli 80125, Italy \\ 2 Dipartimento di Fisiologia Generale e Ambientale, Universita' degli Studi di \\ Napoli 'Federico II', Via Mezzocannone 8, Napoli 80134, Italy \\ 3 These authors contributed equally to the work \\ * Correspondence: S Filosa, Institute of Genetics and Biophysics, 'Adriano \\ Buzzati Traverso', CNR, Via Marconi 10, Napoli 80125, Italy. \\ Tel: + 39-081-7257263; Fax: + 39-081-5936123; \\ E-mail: filosa@iigb.na.cnr.it
}

Received 03.9.03; revised 03.12.03; accepted 23.1.04; published online 26.3.04 Edited by G Melino

\begin{abstract}
Glucose-6-phosphate dehydrogenase-deleted embryonic stem (ES) cells (G6pd $\Delta$ ) proliferate in vitro without special requirements, but when challenged with oxidants fail to sustain glutathione disulphide reconversion to reduced glutathione (GSH), entering a condition of oxidative stress. Here, we investigate the signalling events downstream of GSH oxidation in G6pd $\Delta$ and wild-type (wt) ES cells. We founc that G6pd $\Delta$ ES cells are very sensitive to oxidants, activatit. apoptotic pathway at oxidant concentrations otherwis sublethal for wt ES cells. We show that the apor oth athway activated by low oxidant concentrations is accompal / $/$ by mitochondria dysfunction, and it is there re blocked by the overexpression of $\mathrm{Bcl}-\mathrm{X}_{\mathrm{L}}$. Bcl- $\mathrm{X}_{\mathrm{L}}$ does not hibit th' decrease in cellular GSH and reactive orvaen $s_{p} \ldots . .3$ formation following oxidant treatment. We clso und that oxidant treatment in ES cells is follow- d by the- activation of the MEK/ extracellular signal-regul a od $\mathrm{k}$ ase (L/,K) pathway. Interestingly, ERK activation has uow outcomes in G6pd $\Delta$ ES cells compared to w' "which ha proapoptotic function in the first and a prosur iva nction in the latter. We show that this phenomenon can be reg. ated by the cellular GSH level.

Cell Death. N D'fferentiation (2004) 11, 823-831.

doi:10.1038/s].s 440,1420

Publ shea unline so March 2004
\end{abstract}

Keywo apoptosis; G6PD deficiency; MAP kinases; oxidative stress; Gós

Abbreviations: CisPt, cisplatin; ERK, extracellular signal-regulated kinases; G6PD, glucose 6-phosphate dehydrogenase; Gox, glucose oxidase; GPX, glutathione peroxidase; GSH, reduced glutathione; GSSG, glutathione disulphide; $\mathrm{H}_{2} \mathrm{O}_{2}$, hydrogen peroxide; JNK, c-Jun N-terminal kinases; MAPK, mitogen-activated protein kinases; Mq, menadione; NAC, $\mathrm{N}$-acetyl cysteine; $\mathrm{NADPH}$ reduced nicotinamide adenine dinucleotide; PARP, poly(ADP- ribose) polymerase; PPP, pentose phosphate pathway; ROS, reactive oxygen species; $S O D$, superoxide dismutase

\section{Introduction}

Apoptosis is a genetically programmed fo of cell death that ends in the complete disassemblv of $t_{1}$ ma cellular structures through the activation of a fami of cysteine proteases, the caspases. Activatic of casplases is generally the result of a complex intec tion veath-inducing signals and survival signals, wh re control of mitochondrial integrity has been shoy to play a ntral role. ${ }^{1-3}$ Cell death by apoptosis can be i gge $\checkmark$ by a variety of different stimuli, ranging from a de ${ }^{\cdots}$ opmen ctimulus to the detection of an unsustainable mo it of DNA damage. One of the major threats to the 15 is represented by atmospheric oxygen. Evolution adaptation to life in an oxidant atmosphere $r \in$ nted/a big achievement in the efficiency of bioenergy proau con, but at the same time a serious threat for life itself. $C \in$ lls were required to learn that the oxygen should Do 'ndled with care' in order to avoid irreversible damage to mosi f the cellular components. To be able to cope with the vhl reactive oxygen species (ROS), the main side effect of lit, $n$ an oxidant atmosphere, cells therefore had to evolve an Ifficient antioxidant defence capability. Oxidative stress occurs in the cell when the antioxidant defence cannot keep pace with the rate at which ROS are generated. The response of the cell to oxidative stress can be very different, depending on the intensity of the stress and its duration, and goes from the stimulation of cell proliferation to cell cycle arrest, to cell death by apoptosis or necrosis. ${ }^{4,5}$

The source of oxidant generation can be external to the cell or the side product of normal aerobic metabolism. In all cases, cells respond to the redox imbalance by producing more reducing power. The most abundant and readily available reducing power molecules are glutathione (GSH) and the phosphorylated form of the reduced nicotinamide adenine dinucleotide (NADPH). These two molecules are strictly interdependent, given that glutathione reductase, the enzyme responsible for the recycling of the oxidised form of GSH (glutathione disulphide, GSSG), uses NADPH as a cofactor. NADPH is also used as a cofactor by catalase, another antioxidant enzyme that converts hydrogen peroxide $\left(\mathrm{H}_{2} \mathrm{O}_{2}\right)$ into water and oxygen. ${ }^{6}$

The establishment of a condition of oxidative stress has been implicated in the process of ageing and as the main cause of several human age-related degenerative diseases, including neurodegenerative disorders, like Parkinson and Alzheimer, ${ }^{7}$ the genesis of type II diabetes, ${ }^{8}$ heart failure and stroke. ${ }^{9}$ At the cellular level, production of ROS affects various signal transduction pathways and redox-responsive transcription factors. A large number of mitogen-activated protein kinases (MAPK) signalling pathways are involved in coordinating the response to ROS formation, therefore influencing 
cell destiny. MAPKs encompass a large number of serine/ threonine kinases involved in regulating a wide array of cellular processes including proliferation, differentiation, stress adaptation and apoptosis. ${ }^{10}$ The extracellular signalregulated kinase (ERK), c-Jun $\mathrm{N}$-terminal kinases (JNK) and p38 MAPK subfamilies have all been shown to be activated in response to oxidant injury, and therefore could potentially contribute to influencing survival or apoptosis following oxidative insults. ${ }^{11}$ Although the ERK pathway was initially described to be unresponsive to stress stimuli, it is now clear that oxidative stress can lead to substantial activation of ERK in some cases, and that growth factor receptors play an important role in mediating this effect. A number of studies carried out on a variety of cell types and diverse model agents demonstrated that elevated ERK activity enhanced survival of cells treated with oxidants. However, other studies using different model systems suggest that ERK activation can contribute to apoptosis in response to oxidant injuries. ${ }^{5,12}$

Glucose 6-phosphate dehydrogenase (G6PD) is the first and rate-limiting enzyme of the pentose phosphate pathway (PPP), which catalyses the oxidation of glucose-6-phosphate to glucono- $\delta$-lactone-6-phosphate, concomitantly producing NADPH. This pathway represents an alternative shunt for glucose catabolism downstream of glucose phosphorylation. The choice between glucose oxidation through the glycolitic pathway, or its channelling into the alternative shunt of the PPP, depends on the conditions in which the cell grows, as well as on specific metabolic requirements. ${ }^{6}$ Although mo ace embryos deleted of the G6PD enzyme fail to $d$ velpp normally, ${ }^{13}$ we and others have shown that G6pr-dt embryonic stem (ES) cells can grow withor particu. requirements under normal tissue culture on ans. ${ }^{14,1}$ However, if the cells are challenged with oxidants, th fail to sustain GSSG reconversion to $\mathrm{GSH}$, und rlying a central role for NADPH production in GSH homeost sis. ${ }^{15}$ In this paper, we show that, after oxidant treatment, G6 valced (G6pd $\Delta$ ) ES cells enter into a condition of o> tive stress that strongly increases the level of cellular ROS anc. vates a cell death pathway with all the featı. of a nitochondria-dependent apoptotic pathway. Furt $r$ c a we show that activation of the ERK kinase play a c ral rule in the signalling events downstream of th $3 \mathrm{SH} / \mathrm{GS}$ Y imbalance, establishing the fate of the cell in tern. of life and death.

\section{Results \\ Ta get d delt,cion of the G6pd gene sensitises ES cel. 4 ... apoptotic and necrotic effect of oxida. $y$}

We have recently generated, by homologous recombination, ES cells deleted of the gene coding for G6pd (G6pd $\Delta$ ) and shown that diamide (Dia), a sulphidryl group oxidising agent, drastically decreases G6pd $\Delta$ ES cell viability. ${ }^{15}$ To investigate the features of this loss of viability, wild-type (wt) and G6pd $\Delta$ ES cells were challenged with a 30 min oxidative burst using increasing concentrations of Dia. After $8 \mathrm{~h}$, cells were collected and analysed for markers of apoptosis. As shown in Figure 1a, both caspase-3 and poly(ADP-ribose) polymerase a

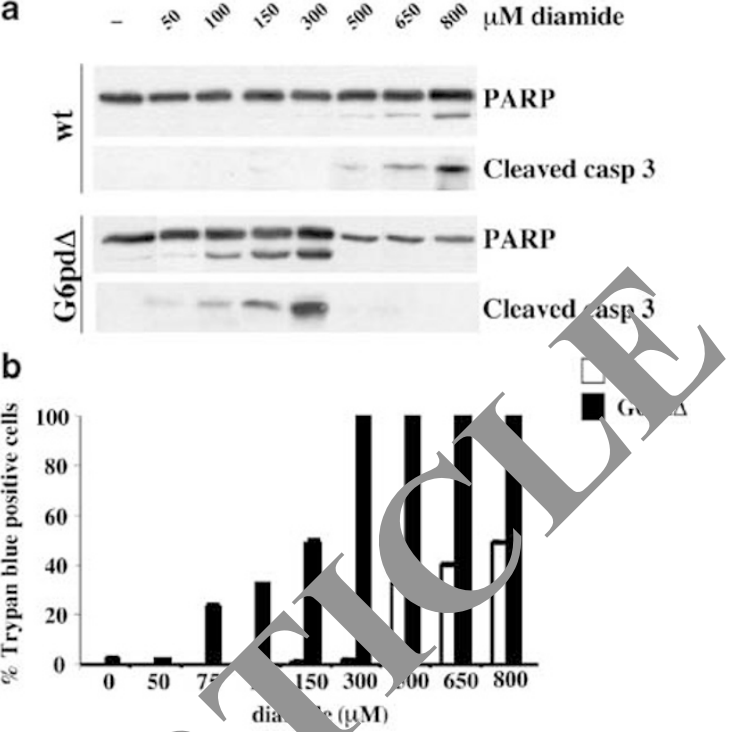

Figure 1 ES cell, deli $d$ of the G6pd gene are extremely sensitive to Dia treatment. (a) Wh $\quad 6 p$ m incubated with different concentrations of Dia for $30 \mathrm{~min}$. After $8 \mathrm{~h}$, ' 'proterns were extracted and separated on SDS-PAGE, and their manective con in cleaved PARP and caspase-3 were analysed by Western blc (h) ES cells, treated as in (a), were analysed for their ability to incorporate the serim imeable dye Trypan blue. The percentage of Trypan bluepositive cells $v$ as calculated over the total number of cells and plotted against the - centration : Dia

(1. ARP) appear to be cleaved at Dia doses as low as $100 \mu \mathrm{M}$ in G6pd-deleted cells. A similar level of caspase-3 activation was evident in wt ES cells only at Dia concentrations eight-fold higher. Interestingly, the amount of caspase-3 and PARP cleavage seemed to be increased by an increased Dia dose, but only up to $300 \mu \mathrm{M}$ concentration (Figure 1a). A further increase had the opposite effect, resulting in poor or null caspase- 3 activation, although the cells completely detached from the cell culture dish and became permeable to the Trypan blue dye (Figure 1b). This suggests that G6pd $\Delta$ ES cells activate an apoptotic pathway if triggered with a relatively low oxidative pulse, but become necrotic if the oxidative power reaches a certain threshold.

Diamide is a thiol oxidant that rapidly oxidises $\mathrm{GSH}$ to GSSG. ${ }^{16}$ This creates a redox imbalance inside the cell, because GSH represents the most abundant source of readily available reducing power. On the other hand, Dia has been shown to interfere with other cellular processes, like initiation of protein synthesis. ${ }^{16,17}$ In order to investigate if the apoptotic effect of low Dia doses on G6pd $\Delta$ ES cells was due to redox imbalance or to other redox-unrelated effects, we studied the effect of the GSH precursor $\mathrm{N}$-acetyl-cysteine (NAC) on the apoptosis induced by Dia, arguing that an increase in the GSH pool would titrate down a possible redox-related Dia effect. As shown in Figure 2, G6pd $\Delta$ ES cells are completely protected from Dia-induced apoptosis in the presence of $1 \mathrm{mM}$ NAC. Moreover, the oxidant sensitivity of G6pd $\Delta$ ES cells was completely reverted to wt levels when they were stably transfected with an expression vector containing the cDNA encoding for mouse G6pd under the control of the B-actin promoter (G6pd $\left.\Delta^{\mathrm{pG} 6 \mathrm{pd}}\right)$ (Figure 2, lane 4). 


\section{The extreme sensitivity to apoptosis of G6pd $\Delta$ ES} cells is specific for oxidative insults

GSH metabolism is intimately linked to the rate of NADPH production, because NADPH is used by glutathione reductase to regenerate GSH from its oxidised form, GSSG. In order to study the importance of G6PD-mediated NADPH production on the cellular protection from redox imbalance, we studied the effect of oxidants, other than Dia, on the viability of G6pd $\Delta$ ES cells. In particular, we focused on $\mathrm{H}_{2} \mathrm{O}_{2}$, by itself an ROS acting at the cytoplasmic level, and on menadione (Mq), a quinone compound that induces the production of mitochondrial superoxide radicals. In order to have more reproducible concentrations of $\mathrm{H}_{2} \mathrm{O}_{2}$ in the cell culture medium, we used glucose oxidase (Gox), an enzyme that produces $\mathrm{H}_{2} \mathrm{O}_{2}$ from glucose and water. As shown in Figure $3, \mathrm{G} 6 \mathrm{pd} \Delta \mathrm{ES}$ cells are extremely sensitive, compared with wt ES cells, to both Gox and Mq. Again, as observed with Dia, this effect was reverted by the reintroduction of G6pd or by the presence of NAC (data not shown).

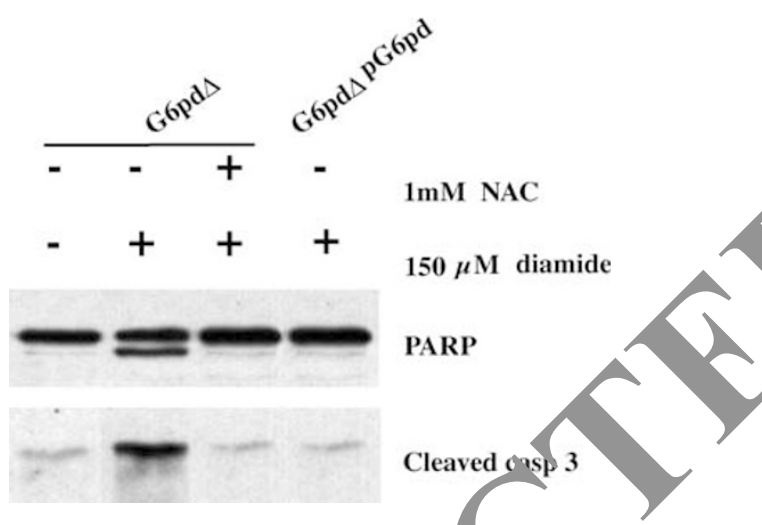

Figure 2 Reintroduction of the G6pd gene or treat ent with the antioxidant NAC protects G6pd $\Delta$ ES cells from the apoptotic e of Dia G6pd $\Delta$ and $\mathrm{G} 6 \mathrm{pd} \Delta^{\mathrm{pG} 6 \mathrm{pd}}$ ES cells were treated for $30 \mathrm{~min}$ with $150 \mu \mathrm{k}$. Ane presence or absence of the antioxidant NAC. After $8 \mathrm{~h}, \mathrm{c}$ re harvested and extracts were analysed by Western blotting for PARP aid $\left(u_{1}, e-3\right.$ cleavage

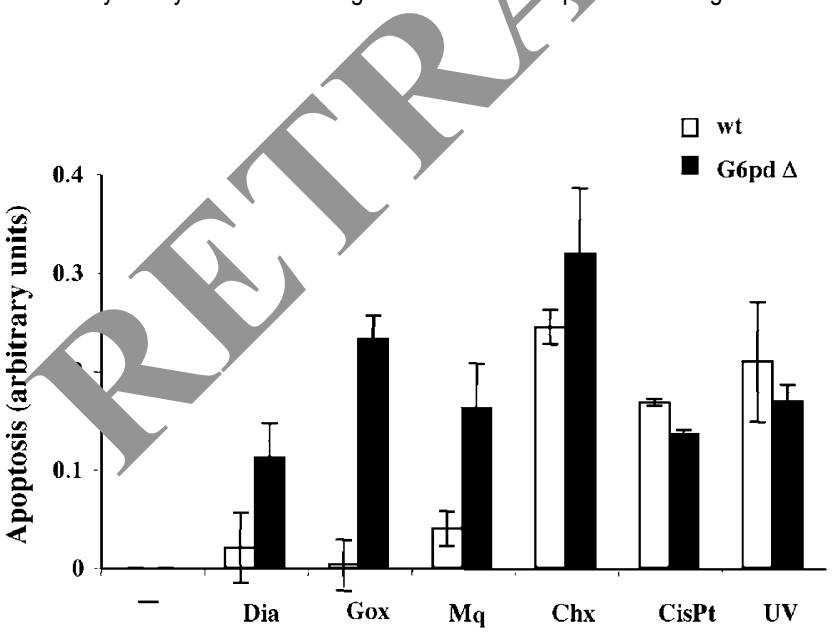

Figure $3 \mathrm{G} 6 \mathrm{pd} \Delta \mathrm{ES}$ cells show an increased sensitivity to the apoptotic effect of oxidants, more than to apoptosis in general. Wt and G6pd $\Delta$ ES cells were treated with Dia, Gox, Mq, Chx, CisPt and UV light as described in Materials and methods. Apoptosis was measured by determining the amount of cytoplasmic DNA-histone complexes using the commercial Cell Death Elisa kit (Roche)
We have shown that G6pd $\Delta$ ES cells undergo programmed cell death when triggered with doses of oxidants otherwise sublethal for wt ES cells. This observation could possibly be explained by invoking a general increased sensitivity to apoptosis given by the absence of G6PD activity. To investigate if G6pd $\Delta$ ES cells show an increased sensitivity to apoptosis, we treated wt and G6pd-deleted ES cells with different apoptotic insults. As shown in Figure 3, the DNAdamaging agents UV and cisplatin (CisPt) induce - a romparable level of cell death in both wt and G6pd-deletec cells A similar result was observed with cycloheximide (C. This was not due to a saturating amount $c$ he agents used, because the same result was obtainer pve. lard range of doses of the tested stimuli (data not shown. These data clearly support a specific role for G PD in the defence against oxidative stress-causing ac ts, hough a role in the protection from apoptoti in 's not tested cannot be excluded.

\section{Diamide-indy cec apoptosis proceeds through a mitochondrica 're ent apoptotic pathway}

In most $f$ the sig it transduction pathways that lead to apoptotic it...th, an early marker of the engagement of the apoptotic rachinery is represented by the release of cutochrome $y$ from the mitochondrial intermembrane space into cytosol. Therefore, we analysed if the apoptotic athv iy triggered by Dia in G6pd $\Delta$ ES cells involved nonondria dysfunction, by studying cytochrome $c$ redistribution following the apoptotic stimulus. Figure 4 a shows that after Dia treatment, G6pd $\Delta$ ES cells release cytochrome $c$ into the cytosol in a time-dependent manner, probably as a consequence of lost mitochondrial permeability. Cytochrome $c$ release was followed by the activation of caspase-9,

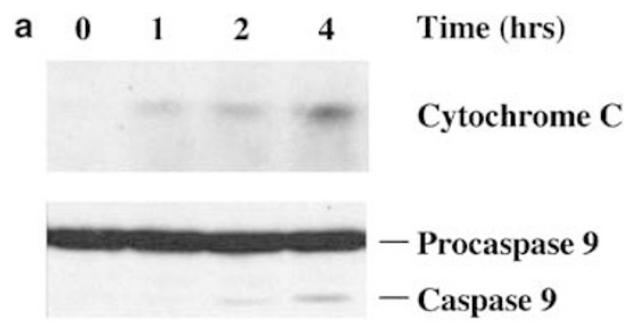

b

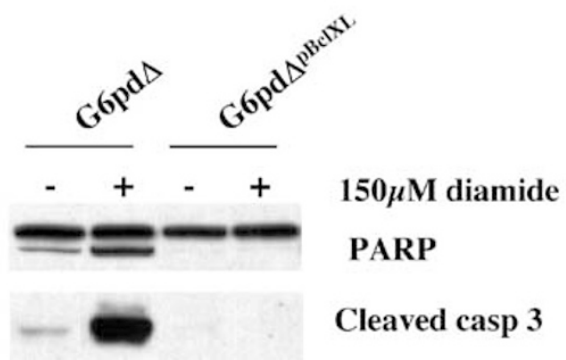

Figure 4 Dia-induced apoptosis in G6pd $\Delta$ ES cells proceeds through the mitochondrial pathway. (a) G6pd $\Delta$ ES cells were treated with $150 \mu \mathrm{M}$ Dia for $30 \mathrm{~min}$ and then collected at the indicated time points. Cytosolic fractions were analysed by Western blot for the presence of cytochrome $c$ or for the cleavage of caspase-9. (b) G6pd $\Delta$ and G6pd $\Delta^{\mathrm{pBClXL}}$ ES cells were treated for $30 \mathrm{~min}$ with $150 \mu \mathrm{M}$ Dia. After $8 \mathrm{~h}$, cells were harvested and extracts were analysed by Western blotting for PARP and caspase-3 cleavage 
presumably through the induction of the apoptosome formation.

$\mathrm{Bcl}-\mathrm{X}_{\mathrm{L}}$ is considered to be a general regulator of mitochondrial homeostasis, and protects from essentially most of the apoptotic triggers. As Dia induced an apparent mitochondria dysfunction, assayed by detecting of cytochrome $c$ release, we addressed the possibility that G6pd $\Delta$ ES stably transfected with an expression vector containing the cDNA encoding for $\mathrm{Bcl}-\mathrm{X}_{\mathrm{L}}$ under the control of the $\beta$-actin promoter $\left(\mathrm{G} 6 \mathrm{pd} \Delta^{\mathrm{Bcl}-\mathrm{X}_{\mathrm{L}}}\right)$ could be protected from oxidant-induced apoptosis. When the cells were triggered with $150 \mu \mathrm{M}$ Dia, Bcl- $\mathrm{X}_{\mathrm{L}}$-expressing $\mathrm{G} 6 \mathrm{pd} \Delta \mathrm{ES}$ cells showed a complete protection from the apoptotic effect of the Dia, as suggested by the absence of caspase-3 and PARP cleavage (Figure $4 b$ ).

\section{Diamide treatment increases cellular ROS in G6pd $\Delta$ ES cells, independent of $B c l-X_{L}$ expression}

We have previously shown that G6pd $\Delta$ ES cells triggered with low doses of Dia fail to re-establish a correct ratio of cellular GSH/GSSG. ${ }^{15}$ GSH metabolism is tightly linked to ROS scavenging. In fact, glutathione peroxidase catalyses the conversion of $\mathrm{H}_{2} \mathrm{O}_{2}$ into water using the reducing power of GSH. Furthermore, other forms of ROS, namely, mitochondrial or cytosolic superoxide anions, can be ultimately neutralised by cellular GSH through their conversion to $\mathrm{H}_{2} \mathrm{O}_{2}$, mediated by superoxide dismutase (SOD) 1 and 2 . Low levels of cellular GSH could therefore be accompanied by an increase in the intracellular concentration of ROS vve made use of ROS-sensitive fluorescent probes to det th ? amount of ROS generated by the treatment with $150, \mathrm{ML}$ of both wt ES cells and G6pd $\Delta$ ES cells, transfe with a expression vector encoding for $\mathrm{Bcl}-\mathrm{X}_{\mathrm{L}}$. As expt $\mathrm{d}$, Dla treatment produced an appreciable increase in cellul. ROS only in G6pd $\Delta$ ES cells (Figure 5). Inte stingly, G6pd $\Delta$ ES cells showed an increased basal ROS co int compared to wt ES cells. Forced expression of $\mathrm{B}-\mathrm{L}-\mathrm{X}_{\mathrm{L}}$ corn, .ely abolished Dia-induced death, but did not $\mathrm{A}_{\mathrm{L}}+$ the production of cellular ROS. Bcl-X, therefore, waid act downstream of ROS production in the ?.pop tic pathway triggered by low

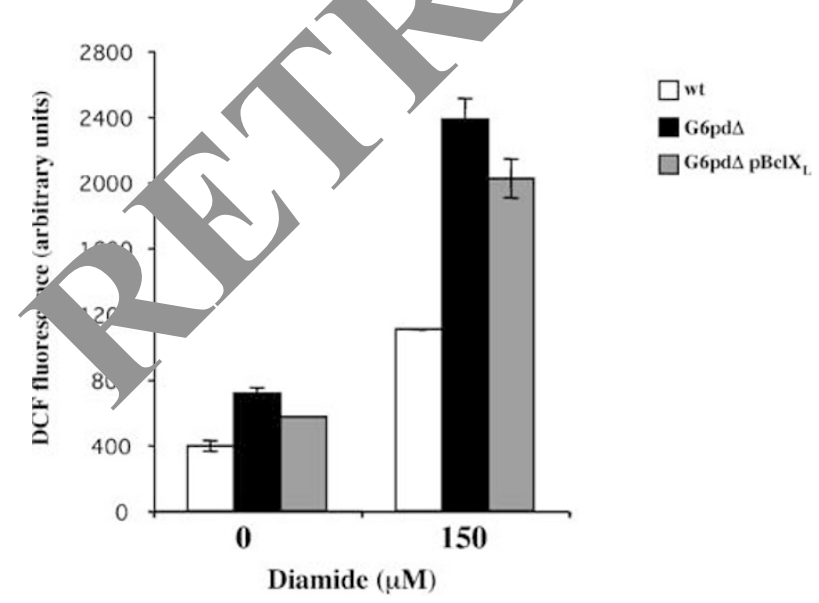

Figure 5 Treatment of G6pd $\Delta$ ES cells with Dia is accompanied by the production of cellular ROS. Production of cellular ROS in the presence of $150 \mu \mathrm{M}$ Dia was quantified after 30 min using the ROS-sensitive fluorescent probe $2^{\prime}, 7^{\prime}$ dichlorofluorescein diacetate (DCFDA) doses of Dia in G6pd $\Delta$ ES cells. In agreement with this, also basal levels of ROS in G6pd $\Delta$ ES cells were not influenced by $\mathrm{Bcl}-\mathrm{X}_{\mathrm{L}}$ expression.

\section{Different from JNK and p38 SAPKs, ERK activation reflects the oxidative stress sensitivity of G6pd $\Delta$ with respect to wt ES cells}

The data presented here suggest that ES "s respond specifically to a proapoptotic signal transduction p. way inat senses a drop in the GSH/GSSG ratio. The amoun t $\mathrm{GSH}$ oxidant needed to reach a GSH/GSS ratio apable of triggering this pathway might cepend o the NADPHproducing capability of the cell Cellular redox imbalance caused by altered GSH metarolis was shown to be sensed, in other cell systems, by soe $\mathrm{Mr}$. . pathways. ${ }^{4,5}$ In order to study the activation status of $1 \mathrm{PK}$ pathways in response to Dia in ES cells used intibodies raised against phosphorylated ERK, JNr nd p38. As expected, treatment of wt and G6pd/ $\mathrm{L}$ cells wI.n different concentrations of Dia markedly res a tho activation of different MAPK pathways (Figure 6). NK $1 / 2$ were found to be phosphorylated using 51 M Dia Ir G6pd $\Delta$ and $100 \mu \mathrm{M}$ Dia in wt ES cells. Similarly, ho how exactly the same activation profile as JNK1/2 in wt and G6pd $\Delta$ ES cells. As shown in Figure 1a, "unse two xidant concentrations do not correlate with the inc tion of apoptosis either in G6pd $\Delta$ or in wt ES cells Interestingly, ERK $1 / 2$ activation profile was strikingly frent between wt and G6pd $\Delta$ ES cells. In wt cells, prosphorylated ERK $1 / 2$ could be detected at $500 \mu \mathrm{M}$ Dia, while in G6pd $\Delta$ ES cells, a 10-fold lower concentration of Dia was sufficient (Figure 6). Therefore, ERK activation reflected the difference in oxidative stress sensitivity between wt and G6pd $\Delta$ ES cells.

\section{Inhibition of MEK/ERK activation decreases Dia- induced apoptosis in G6pd $\Delta$, but not wt in ES cells}

Activation of MAPK pathways following oxidative stress has been shown to produce different outputs. According to the intensity and persistence of the stimulus, they can either increase the survival potential of cells by inducing the transcription of several antioxidant enzymes or determine cell death through mechanisms in part still to be elucidated. ${ }^{4,5}$

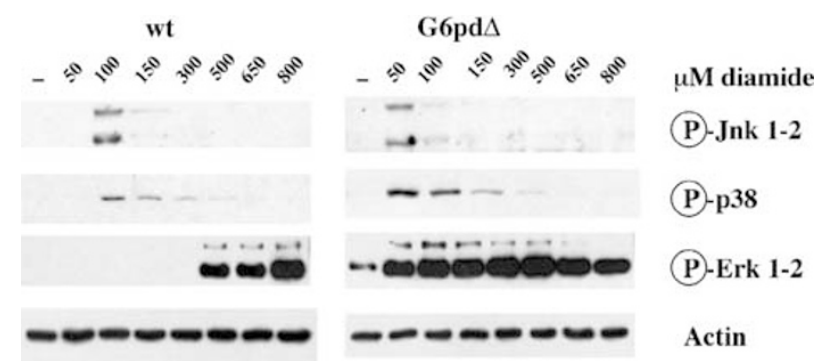

Figure 6 Analysis of JNK1/2, p38 and ERK $1 / 2$ following Dia treatment in Wt and G6pd $\Delta$ ES cells. Wt and G6pd $\Delta$ ES cells were incubated for 30 min with the indicated amount of Dia. Total cell lysates were prepared and analysed by Western blotting using antibodies against the phosphorylated forms of JNK1/2, p38 and ERK1/2. The analysis of actin content was used as a loading control 
The pattern of ERK activation suggested a possible role for this kinase in the induction of apoptosis. To address this hypothesis, we made use of U0126, an inhibitor of the activation of the upstream kinase MEK, thus abolishing ERK phosphorylation. Wt and G6pd $\Delta$ ES cells were treated with a $30 \mathrm{~min}$ Dia pulse at the doses known to induce apoptosis (see above) in the presence or absence of the MEK inhibitor U0126. Cells were then analysed either after $30 \mathrm{~min}$, to detect ERK phosphorylation, or after $8 \mathrm{~h}$, to investigate PARP and the caspase-3 cleavage status, as a marker for apoptotic cell death. As shown in Figure 7a, treatment with U0126 while completely inhibiting ERK phosphorylation in both cell lines, had remarkably the opposite effect on apoptosis markers in wt and $\mathrm{G} 6 \mathrm{pd} \Delta \mathrm{ES}$ cells. When we treated wt ES cells with a lethal dose of Dia, in the presence of the MEK inhibitor, we could reproducibly observe an increased amount of the faster migrating PARP cleavage product, as well as an increase in the amount of activated caspase 3, suggesting a role of MEK/ ERK in an antiapoptotic pathway. On the contrary, in G6pd $\Delta$ ES cells, the presence of U0126 strongly decreased the level of activated caspase- 3 and cleavage of PARP, as well as the release of cytochrome $c$ from mitochondria and the activation of caspase-9 (Figure 7b), suggesting an active role for the MEK/ERK pathway in mediating the apoptotic effect of Dia.

These results, therefore, show that MEK/ERK activation plays a proapoptotic role in the G6pd $\Delta$ ES cells, while in the wt cell lines, ERK activation is either insufficient or possibly implicated in an antiapoptotic response.

\section{ERK activation has multiple roles in cell fate decisions after Dia treatment}

The observation that G6pd $\Delta$ ES cells activated different MAPK pathways in response to oxidative stress with respect to the parental wt ES cells was, in part, expected, given the strong difference in apoptosis sensitivity of the two cell lines. The results shown in Figure 7, though, suggest that the absence of G6PD does not simply lower the thresnold for oxidative stress sensitivity. Diamide concer. tio's that induce apoptotic cell death in wt and G6pd $\Delta$ ES VIIs y, th similar kinetics (data not shown) activate the same $p$.nway with opposite outputs. One possibility, to e, ain this apparent discrepancy, is that a change in GSH'GSSG is sensed by the MEK/ERK pathway in ES cells and that the effect of this activation, in terms of life or dea for the cell, could then depend on the persistence o st as well as from the intensity. We have alrea y sho ${ }^{15}$ that after $30 \mathrm{~min}$ of Dia treatment, most of the $c t$ ar GSH,s converted to GSSG. To investigate whether stiess sistence might play a role in the output of MEK/F-1. activatio, 1 , we treated $\mathrm{G} 6 \mathrm{pd} \Delta \mathrm{ES}$ cells with shorter p' so 450 M Dia. As shown in Figure 8 (lanes 1 and 2), a 10 . Dia pulse was already able to induce caspase- activatio, and PARP cleavage in G6pd $\Delta$ ES cells. Strikingly, vine analysed the role of the MEK/ERK pathway $b$ ' treating the cells with U0126 during the two diffsrent Dia , Julses (Figure 8, compare lanes 2/3 and 4/5), we obs ed that U0126 treatment increased the level of asp e -3 activation at the 10 min incubation time and 1. ried its effect to an inhibitor of caspase-3 activation, as already shown in Figure 7, at the 30 min incubation time point, suggesting that ES cells try to rescue the death induced by the change in GSH/GSSG ratio by activating an MEK/ERKdependent survival pathway, but if the GSH oxidation persist, they convert the MEK/ERK-dependent survival pathway into a proapoptotic one.

\section{Diamide treatment induces the formation of GSSG in G6pd $\Delta$ but not in wt ES cells}

The results shown in Figures 6 and 7 suggest that, although 800 and $150 \mu \mathrm{M}$ Dia kill wt and G6pd $\Delta$ ES cells with similar kinetics, respectively, they probably induce cell death through different signal transduction pathways. This difference could

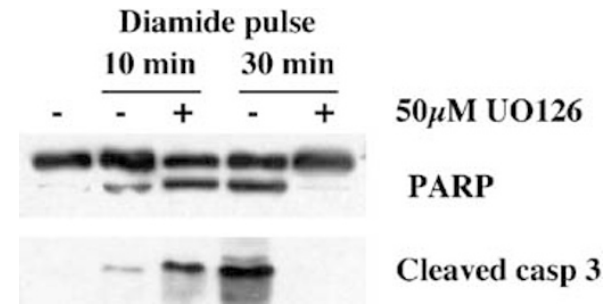

Figure 8 Inhibition of Dia-induced ERK activation in G6pd $\Delta$ ES cells has different outputs, depending on the duration of the stress. The effect of the MEK inhibitor U0126 was analysed on the apoptosis induced by $150 \mu \mathrm{M}$ Dia on G6pd $\Delta$ ES cells. Cells were treated for 10 and 30 min with $150 \mu \mathrm{M}$ Dia in the presence or absence of the MEK inhibitor U0126 and collected after $8 \mathrm{~h}$ to measure the cleavage status of PARP and caspase-3. The figure is a Western blot analysis using antibodies specific for PARP or for the cleaved form of caspase-3 
reflect a difference in the amount of GSH oxidised or in its oxidation rate in wt and $\mathrm{G} 6 \mathrm{pd} \Delta \mathrm{ES}$ cells treated with the respective lethal doses of Dia. We therefore measured the kinetics of GSH decrease and GSSG appearance in wt and G6pd $\Delta$ ES cells upon incubation with 800 and $150 \mu \mathrm{M}$ Dia, respectively. As shown in Figure 9, after $10 \mathrm{~min}$ of Dia incubation there was already a dramatic drop in the GSH cell content both in wt ES cells treated with $800 \mu \mathrm{M}$ Dia and in $\mathrm{G} 6 \mathrm{pd} \Delta \mathrm{ES}$ cells treated with $150 \mu \mathrm{M}$ Dia, although the level of decrease was much more pronounced in the cells devoid of G6PD activity. Analysis of GSSG cell content during the time after Dia treatment also revealed a very different picture between wt and G6pd $\Delta$ ES cells: we were unable to detect any traces of GSSG in wt ES cells even after 30 min of incubation with $800 \mu \mathrm{M}$ Dia. GSSG, instead, reached its maximum level in G6pd $\Delta$ ES cells already after 10 min of $150 \mu \mathrm{M}$ Dia treatment, suggesting that the conditions of oxidative stress induced in wt and G6pd $\Delta$ ES cells upon incubation with 800 and $150 \mu \mathrm{M}$ Dia, respectively, have strongly different features.

\section{Discussion}

By targeting the gene coding for G6PD in mouse ES cells for deletion, we have previously shown that the reducing potential produced by G6PD in the form of NADPH is essential for an efficient response to oxidant treatment. G6pd-deleted ES cells fail to sustain high GSH levels when treated with low levels of oxidating agents. In this paper, we analyse the signalling events downstream of GSH oxidation in terr 15 of apoptosis and/or survival in G6pd $\Delta$ ES cells in compa $n$, wt ES cells. Furthermore, we investigate if the cronditic oxidative stress that we observe at low oxidant cs, ontration. in G6pd $\Delta$ ES cells are equivalent to the o, idat stress induced by high oxidant doses in ES cells wt tor G6PL

We have previously reported that G6p $\Delta$ ES cells become permeable to Trypan blue staining afte reatment with Dia doses otherwise sublethal for $w^{+}$ES ce...15 Trypan blue permeability could be the conseque ther of apoptotic cell death or necrotic cell death. It has bee: shown, in different cell systems, that oxidant tre.tme , if no' counterbalanced by an

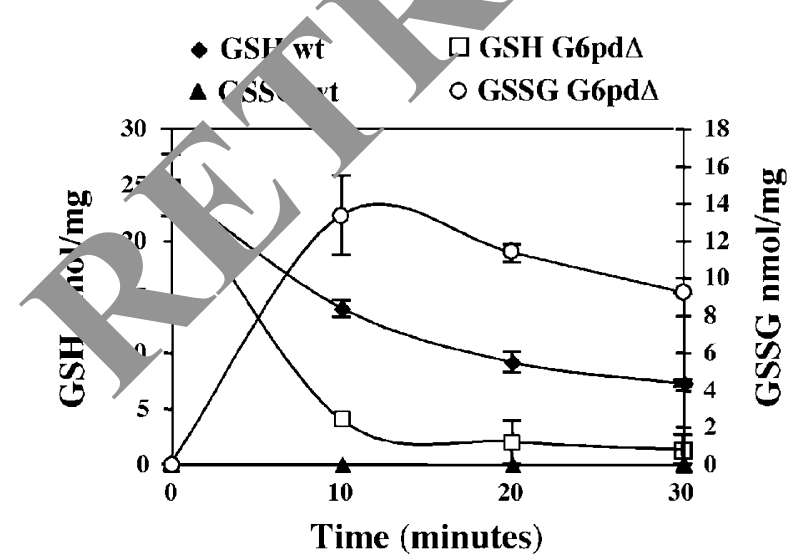

Figure 9 Contrary to what was observed in G6pd $\Delta$ ES cells, GSH decrease in Dia-treated wt ES cells is not accompanied by a GSSG increase. Wt and G6pd $\Delta$ ES cells were treated for the indicated amount of time with 800 and $150 \mu \mathrm{M} \mathrm{Dia}$, respectively. After acidic extraction, changes in cellular GSH and GSSG content were analysed by HPLC appropriate antioxidant response, can induce either an apoptotic pathway or necrosis. The decision between apoptosis and necrosis is usually dictated by the strength of the oxidative insult. ${ }^{18}$ Accordingly, we found that low doses of Dia engage an apoptotic pathway in G6pd $\Delta$ ES cells, as measured by the appearance of the cleaved forms of caspase-3 and PARP. By increasing the Dia concentration over $300 \mu \mathrm{M}$, all cells became Trypan blue positi s without caspase-3 activation, a condition that is che acteristic of necrotic cell death. Interestingly, at these Dia co, Irati ns, the control wt ES cells did not show any signs of nase-3 activation or permeability to Trypan blu to indure a similar level of caspase-3 activation in wt EC cells, was necessary to increase Dia concentration up to eight tir is more. This increased sensitivity of G6pd-dt ted ES/cells against the effect of Dia is likely due + the + ox mbalance that we previously observed in thr se characterised by low GSH and low NADPH conte becaus re effect can be reversed by treating the cells vith GSH precursor, NAC.

The establishment of a c dition of redox imbalance was followed by $d f$ stab, sation of the outer mitochondrial permeability that pre apidly triggered the apoptotic pathway. We could ir ad completely block caspases activation by overe - aing ine antiapoptotic protein Bcl- $\mathrm{X}_{\mathrm{L}}$ in $\mathrm{G} 6 \mathrm{pd} \Delta$ ES cells. $O=L_{1}<$ other hand, $\mathrm{Bcl}-\mathrm{X}_{\mathrm{L}}$ expression prevented ROS formction following Dia treatment only to a very small c. nt (Figure 5), suggesting a role in blocking the apoptotic sign. transduction pathway rather than in preventing oxidave tress. In line with this, also the decrease in GSH levels a $d$ ERK activation (see below) was not influenced by Bcl- $X_{L}$ expression (data not shown). If $\mathrm{Bcl}-\mathrm{X}_{\mathrm{L}}$ expression does not prevent oxidative stress, but only the activation of the apoptotic pathway, this would imply that life is compatible with a certain amount of oxidation of cellular structures. Otherwise $\mathrm{Bcl}-\mathrm{X}_{\mathrm{L}}$ expression, similar to what has been observed with $\mathrm{Bcl}-2,{ }^{19}$ might play a role also as an antioxidant, by blocking the damaging effects of peroxides, rather than preventing their formation.

Although a possible involvement of G6PD in the protection from apoptosis was previously indirectly suggested by the use of the G6PD inhibitors dehydroepiandrosterone and 6aminonicotinamide ${ }^{20}$ to our knowledge for the first time a role for G6PD in the protection from redox imbalance-induced apoptosis and necrosis has been clearly assigned, also in view of the complex and multiple physiological effects triggered by these inhibitors. ${ }^{21}$ That G6PD could be a mediator of a survival pathway was also previously suggested by the fact that G6PD overexpression in HeLa cells increased their resistance to $\mathrm{H}_{2} \mathrm{O}_{2}$ and TNF $\alpha$ treatment. ${ }^{22}$

G6PD is not the only NADPH-producing enzyme in nucleated cells, although we have previously shown that it is the only one strongly activated by oxidant treatment. Probably, the peculiarity of G6PD lies in the fact that it uses a highly abundant and readily available source of reducing power, glucose, and this makes it the rate-limiting factor in antioxidant defence. This should be taken into account when considering the importance of glucose in cell survival. In the absence of glucose, in fact, cells are not only unable to proceed through the glycolytic pathway but they are also affected in the production of the main reducing equivalent, 
NADPH. For instance, the oncogene Akt has a broad antiapoptotic activity but, different from Bcl- $\mathrm{X}_{\mathrm{L}}$, strictly requires glucose availability to afford protection against apoptosis. $^{23,24}$ It has been suggested that glucose phosphorylation is sufficient to promote cell survival by Akt, based on the fact that 2-deoxyglucose, a glucose analogue that can be phosphorylated by hexokinase but not further metabolised through the glycolytic pathway, restores Akt ability to prevent apoptosis. ${ }^{23}$. Given the importance for G6PD activity in cell survival and given that 2-deoxyglucose-6-phosphate, although not further metabolised during glycolysis, is accepted as a substrate by $\mathrm{G} 6 \mathrm{PD},{ }^{25}$ we propose that the strict glucose requirement of Akt-induced survival could depend on the activity of the PPP shunt.

Although G6PD seems to have a central role in protection from apoptosis, this effect seems to be specific for apoptosis induced by oxidating agents. G6pd-deleted ES cells were equally sensitive to Chx-induced cell death (Figure 3) and serum starvation (data not shown), and even slightly more resistant to the death induced by UV irradiation or CisPt. On the other hand, G6PD-deleted cells showed increased sensitivity to agents inducing, directly or indirectly, ROS, and ROS production has been suggested to be and intermediated in several apoptotic pathways. ${ }^{4}$ It will be interesting to investigate if G6pd-deleted cells could also be sensitive to conditions accompanied by physiological levels of intracellular ROS production, as it has been suggested to occur during TNF $\alpha$ treatment or prolonged p53 activation. ${ }^{26-28}$ We are currently deriving an epithelial-like $r$ N. line from in vitro differentiated ES cells to addresc this possibility.

In order to study the signal transduction pathw? activate by Dia-induced oxidative stress in ES cells de tec r not of the G6pd gene, we investigated the phosphor, 'ation s. is of the major cellular MAPK pathways. The pa hway that mikrored most closely the difference in oxidative str sensit vity found in wt and G6pd $\Delta$ ES cells was the $\mathrm{Mt}$. 'CR' pathway. Interestingly, MEK/ERK activation s med to have a multiple

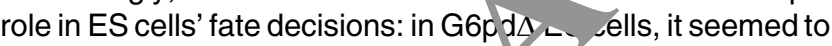
have a protective role follor... a ver short pulse of oxidant treatment, and clearly roe nntotic function following a longer oxidant exposy In ES cells, on the contrary, MEK/ ERK activation ser ad to ha a protective role in all the conditions teste. In pendent of its role in apoptosis induction, ov data cle indicate that in ES cells the establishm it of a condition of redox imbalance is sensed by the MEK/L K pat/way. Although JNK and p38 were also activare t difte it oxidant concentrations in wt and G6pd $\Delta$ ES $1 / s$ mir activation was transient and did not correlate with $r_{t} x$ imbalance and apoptosis induction. We observed in fact, $\checkmark$ and p38 activation in wt ES cells treated with $100 \mu \mathrm{M}$ Dia, a condition that does not induce apoptosis (Figure 1). How does oxidative stress activate MEK/ERK? According to a previously reported study, a possible explanation involves the inactivation, through disulphide bonds formation, of receptor tyrosine phosphatases. These keep normally the activation level of receptor tyrosine kinases low, following prolonged stimulation by receptor ligands. Transient inactivation of the phosphatases results therefore in transient activation of the kinases receptor. ${ }^{29}$ Receptor tyrosine kinases are among the strongest activators of the MEK/ERK pathway. Indeed, we have preliminary results showing a general increase in phosphotyrosines following Dia treatment (data not shown).

As mentioned above, MEK/ERK activation produces opposite outputs, depending on the persistence of the stress and on the G6PD status of the cells. The fact that a $10 \mathrm{~min}$ pulse of $150 \mu \mathrm{M}$ Dia in G6pd $\Delta \mathrm{ES}$ cells results in the tivation of the pathway with an apparent antiapoptr ic function (Figure 8) resembled the condition that we obs -ain $N t$ ES cells when treated for $30 \mathrm{~min}$ with $800 \mu \mathrm{M}$ Dia ( 1 re 7 ). This was initially suggesting that, if $t$. e was sinnply a threshold difference between wt and G' $\mathrm{nd} \Delta$ cell $s$, this was higher than initially thought, and also dept dent on the oxidant exposure time. It would possible to extrapolate from this argument that in wt cel ats d for a longer time or with a higher Dia doss, the ctivation of the MEK/ERK pathway would become oapopto ás well. We were unable to confirm this predic ion sause of the necrotic effect that Dia has at higher concentratı and exposure times (data not shown). This $\mathrm{o}^{\mathrm{l}}$ sen tion highlights the difference in oxidant sensitivity betw a... $d$ G6pd $\Delta$ ES cells. This difference became even mort rident when we measured the kinetics of $\mathrm{GSH}$ decl after, reating wt and G6pd $\Delta$ ES cells with their respective le $e^{+}$lai cose of oxidant. We found that not only was there a difference in the kinetics of GSH decrease between II. wo cell lines, but, while the GSH decrease was acco banied by an increase in GSSG level in G6pd $\Delta$ ES I/s, we could not observe any traces of GSSG in wt cells fo, swing oxidant treatment. It is possible to speculate that Jia-treated wt ES cells might use protein thiols instead of a second molecule of GSH as an electron donor and might result in an increased cellular content of glutathionylated proteins, ${ }^{30,31}$ more than GSSG. This observation suggests that we cannot explain the difference in oxidant sensitivity of wt and G6pd $\Delta$ ES cells with a simple threshold model, but we have to consider the conditions of oxidative stress induced by Dia in ES cells, in the presence or absence of a G6PD activity, qualitatively very different.

The results presented in this paper indicate G6PD as one of the major enzymes involved in cell survival following redox imbalance, and a key player in cellular antioxidant defence. In addition, these findings could be particularly relevant in relation to the fact that G6PD deficiency is one of the most common enzymopathies in the human population. ${ }^{32,33}$ As the establishment of a condition of oxidative stress has been implicated as the main cause of several degenerative diseases, including neurodegenerative disorders, like Parkinson and Alzheimer, ${ }^{7}$ the genesis of type II diabetes, ${ }^{8}$ heart failure and stroke, ${ }^{9}$ it will be of great interest to study possible connections between the risk of progression of such diseases and inherited G6PD deficiency.

\section{Materials and Methods}

\section{Reagents and cell culture}

NAC, GSH, Mq, Dia, Chx, CisPt and Gox were purchased from SigmaAldrich. U0126 was from Promega. Antibodies specific for PARP, cleaved caspase-3 (Asp175), caspase-9, phospho-SAPK/JNK (Thr183/Tyr185), 
phospho-p38 MAP kinase (Thr180/Tyr182) and phospho-p44/42 MAP kinase (Thr202/Tyr204) (phospho-ERK1/2) were purchased from New England Biolabs. Antibodies specific for cytochrome $c$ were from $B D$ Biosciences (clone $7 \mathrm{H} 8.2 \mathrm{C} 12$ ). ES cells were cultured on mouse fibroblasts as described by Robertson. ${ }^{34}$

\section{Cell death assay}

At $48 \mathrm{~h}$ before treatment, cells were seeded at equal density on gelatincoated plates. Cells were treated either with Dia, Mq, Gox or Chx for $30 \mathrm{~min}$ and than harvested after $8 \mathrm{~h}$, or with $\mathrm{CisPt}$ for $16 \mathrm{~h}$, or they were UV irradiated at $10 \mathrm{~mJ} / \mathrm{cm}^{2}$ using a Stratalinker 2400 (Stratagene) and collected after $8 \mathrm{~h}$. Where NAC or U0126 was used, cells were first preincubated for $30 \mathrm{~min}$ and then treated as indicated in the presence of the same concentration of the NAC or U0126. For apoptosis assay, cell extracts were either analysed by Western blotting for PARP and caspase3 cleavage or using the commercial kit Cell Death Elisa (Roche).

\section{Western blotting}

Total cell lysates were prepared from cells grown on gelatin-coated plates. Cells were lysed in $10 \mathrm{mM}$ Tris-HCl pH7.4, $150 \mathrm{mM} \mathrm{NaCl}, 1 \mathrm{mM}$ EDTA, 1\% Triton X-100, 10\% glycerol, supplemented with $0.5 \mathrm{mM}$ DTT, $0.5 \mathrm{mM}$ phenylmethylsulphonyl fluoride, $2 \mathrm{mM}$ benzamidine, $20 \mathrm{mg} / \mathrm{ml}$ aprotinin, $4 \mathrm{mg} / \mathrm{ml}$ pepstatin, $10 \mathrm{mM}$ leupeptin, $10 \mathrm{mM}$ sodium fluoride, $1 \mathrm{mM}$ sodium orthovanadate and $25 \mathrm{mM} \beta$-glycerophosphate (Sigma-Aldrich). After 15 min incubation on ice, the lysates were cleared by centrifugation. Totai cell extracts were separated on $10 \%$ or $12.5 \%$ SDS-PAGE and transfe ed to Immobilon-P transfer membranes (Millipore). After blocking y th $5 \%$ nonfat milk, membranes were incubated with primary antibou. recommended by the supplier. The immune complexes we detectea the ECL detection system according to the manuf ctur protocol (Amersham Bioscience).

\section{Detection of cytochrome $c$ release}

To evaluate the amount of cytochrome $c$ rinto cytosol, cells were trypsinised, washed in PBS and resuspende $\mathrm{d}$ in $3,0 \mu \mathrm{l}$ of isotonic-sucrose buffer (20 mM HEPES/NaOH $\mathrm{pH}$, ), $250 \mathrm{nM}$ sucrose, $1.5 \mathrm{mM} \mathrm{MgCl}$, $10 \mathrm{mM} \mathrm{KCl}, 1 \mathrm{mM}$ EDTA $\quad d \times$ Complete $^{\text {TM }}$ (Roche)) containing $5 \mathrm{mg}$ of digitonin $/ 4 \times{ }^{6}$ cells. After incubation for $3 \mathrm{~min}$ at room temperature, ere centi, uged at $9500 \times g$ for $5 \mathrm{~min}$ at $4^{\circ} \mathrm{C}$. The resulting supernatant then separated on $12.5 \%$ SDS-PAGE, and analysed by 'vestern blottin, using a monoclonal antibody specific for cytochrome

Ge. of G6pd and Bcl-X $\mathrm{L}_{\mathrm{L}}$ overexpressing ES cells

For the generation of G6pd and Bcl-X ES cells, the wt and G6pd $\Delta$ ES cell lines were electroporated with $20 \mu \mathrm{g}$ of linearised plasmid vector Pallino $\beta$ actin G6pd or Pallino $\beta$-actin $\mathrm{Bcl}-\mathrm{X}_{\mathrm{L}}$, a plasmid vector containing, respectively, G6pd or $\mathrm{Bcl}-\mathrm{X}_{\mathrm{L}} \mathrm{CDNA}$ driven by $\beta$-actin promoter, and the puromycin-resistance gene driven by the mouse phosphoglycerokinase promoter. Transfected clones were selected by growth in the presence of puromycin $1 \mu \mathrm{g} / \mathrm{ml}$, and the resistant clones were subjected to immunoblot analysis by using anti-rabbit-G6PD antibody (ABCAM) or anti-mouse-Bcl$X_{L}$ antibody (BD Biosciences).

\section{Fluorescent measurement of intracellular ROSs}

For the fluorimetric measure of ROS, cells were trypsinised and washed three times with PBS; $1 \times 10^{6}$ cells were suspended in $2 \mathrm{ml}$ of PBS and incubated with $10 \mathrm{mM} 6$-carboxy-2',7'-dichlorodihydrofluorescein diacetate, di(acetoxymethyl ester) (DCHF-DA) (Molecular Probes), for $20 \mathrm{~min}$ at $37^{\circ} \mathrm{C}$. Then, the cells were washed twice with PBS, suspended in $2 \mathrm{ml}$ of PBS and fluorescence levels were measured using a spectrofluorimeter (FP-777 Jasco) with excitation and emission wavelengths set at 495 and $530 \mathrm{~nm}$, respectively.

\section{GSH and GSSG measurements}

To determine intracellular levels of oxirised and uned glutathione (GSH), acidic extracts from cells untre ted or treated as described in Figure 9 were subjected to revorsec ase hish-performance liquid chromatography using an electroc . ical un vion system (HPLC/ECD) as described. ${ }^{15}$

\section{Acknowledy nents}

We thank J Down ar an the pancor, D De Cesare and L slino for support and advice, C Rallo, M Petrillo and S Cos holp with the computer work and C Sole, M Terracciano and R Vito trineli skilful laboratory assistance. This work was supported bv the Italia ? Telethon Foundation, Grant No. 324/bi and Progetto Iv. ST-CNR Legge 488/92 (Cluster C02).

\section{Keferences}

1. Cory S and Adams JM (2002) The Bcl2 family: regulators of the cellular life-ordeath switch. Nat. Rev. Cancer. 2: 647-656

2. Gross A, McDonnell JM and Korsmeyer SJ (1999) BCL-2 family members and the mitochondria in apoptosis. Genes Dev. 13: 1899-1911

3. Vander Heiden MG and Thompson CB (1999) Bcl-2 proteins: regulators of apoptosis or of mitochondrial homeostasis? Nat. Cell Biol. 1: E209-216

4. Finkel T (2000) Redox-dependent signal transduction. FEBS Lett. 476: 52-54

5. Martindale JL and Holbrook NJ (2002) Cellular response to oxidative stress: signaling for suicide and survival. J. Cell. Physiol. 192: 1-15

6. Stryer L (1995) Biochemistry. New York: W.H. Freeman and Company

7. Lotharius $J$ and Brundin $P$ (2002) Pathogenesis of Parkinson's disease: dopamine, vesicles and alpha-synuclein. Nat. Rev. Neurosci. 3: 932-942

8. Brownlee M (2001) Biochemistry and molecular cell biology of diabetic complications. Nature 414: 813-820

9. Behl C and Moosmann B (2002) Oxidative nerve cell death in Alzheimer's disease and stroke: antioxidants as neuroprotective compounds. Biol. Chem. 383: 521-536

10. Kolch W. (2000) Meaningful relationships: the regulation of the Ras/Raf/MEK/ ERK pathway by protein interactions. Biochem. J. 351 (Part 2): 289-305

11. Johnson GL and Lapadat $R$ (2002) Mitogen-activated protein kinase pathways mediated by ERK, JNK, and p38 protein kinases. Science 298: 1911-1912

12. Cuda G, Paterno R, Ceravolo R, Candigliota M, Perrotti N, Perticone F, Faniello MC, Schepis F, Ruocco A, Mele E, Cassano S, Bifulco M, Santillo M and Avvedimento EV (2002) Protection of human endothelial cells from oxidative stress: role of Ras-ERK1/2 signaling. Circulation 105: 968-974

13. Longo L, Vanegas OC, Patel M, Rosti V, Li H, Waka J, Merghoub T, Pandolfi PP, Notaro R, Manova K and Luzzatto L (2002) Maternally transmitted severe glucose 6-phosphate dehydrogenase deficiency is an embryonic lethal. EMBO J. 21: 4229-4239

14. Pandolfi PP, Sonati F, Rivi R, Mason P, Grosveld F and Luzzatto L (1995) Targeted disruption of the housekeeping gene encoding glucose 6-phosphate dehydrogenase (G6PD): G6PD is dispensable for pentose synthesis but essential for defense against oxidative stress. EMBO J. 14: 5209-5215 
15. Filosa S, Fico A, Paglialunga F, Balestrieri M, Crooke A, Verde $P$, Abrescia $P$, Bautista JM and Martini G (2003) Failure to increase glucose consumption through the pentose-phosphate pathway results in the death of glucose-6phosphate dehydrogenase gene-deleted mouse embryonic stem cells subjected to oxidative stress. Biochem. J. 370: 935-943

16. Kosower NS and Kosower EM (1995) Diamide: an oxidant probe for thiols Methods Enzymol. 251: 123-133

17. Zehavi-Willner T, Kosower EM, Hunt T and Kosower NS. (1971) Glutathione. V. The effects of the thiol-oxidizing agent diamide on initiation and translation in rabbit reticulocytes. Biochim. Biophys. Acta 228: 245-251

18. Gardner AM, Xu FH, Fady C, Jacoby FJ, Duffey DC, Tu Y and Lichtenstein A (1997) Apoptotic vs. nonapoptotic cytotoxicity induced by hydrogen peroxide. Free Radic. Biol. Med. 22: 73-83

19. Hockenbery DM, Oltvai ZN, Yin XM, Milliman CL and Korsmeyer SJ (1993) Bcl2 functions in an antioxidant pathway to prevent apoptosis. Cell 75: 241-251

20. Tian WN, Braunstein LD, Apse K, Pang J, Rose M, Tian X and Stanton RC (1999) Importance of glucose-6-phosphate dehydrogenase activity in cell death. Am. J. Physiol. 276: C1121-1131

21. Gordon GB, Shantz LM and Talalay P (1987) Modulation of growth, differentiation and carcinogenesis by dehydroepiandrosterone. Adv. Enzyme Regul. 26: 355-382

22. Salvemini F, Franze A, lervolino A, Filosa S, Salzano S and Ursini MV (1999) Enhanced glutathione levels and oxidoresistance mediated by increased glucose-6-phosphate dehydrogenase expression. J. Biol. Chem. 274: 2750-2757

23. Gottlob K, Majewski N, Kennedy S, Kandel E, Robey RB and Hay N (2001) Inhibition of early apoptotic events by Akt/PKB is dependent on the first committed step of glycolysis and mitochondrial hexokinase. Genes Dev. 15: 1406-1418

24. Plas DR, Talapatra S, Edinger AL, Rathmell JC and Thompson CB (2001) Akt and $\mathrm{Bcl}-\mathrm{xL}$ promote growth factor-independent survival through distinct effects on mitochondrial physiology. J. Biol. Chem. 276: 12041-12048
25. Le Goffe C, Vallette G, Jarry A, Bou-Hanna C and Laboisse CL (1999) The in vitro manipulation of carbohydrate metabolism: a new strategy for deciphering the cellular defence mechanisms against nitric oxide attack. Biochem. J. 344 (Part 3): 643-648

26. Flohe L, Brigelius-Flohe R, Saliou C, Traber MG and Packer L (1997) Redox regulation of NF-kappa B activation. Free Radic. Biol. Med. 22: $1115-1126$

27. Polyak K, Xia Y, Zweier JL, Kinzler KW and Vogelstein B (1997) A model for p53-induced apoptosis. Nature 389: 300-305

28. Schreck R, Rieber P and Baeuerle PA (1991) Reactive oxygf intermediates as apparently widely used messengers in the activation or N kappa B transcription factor and HIV-1. EMBO J. 10: 2247-2258

29. Blanchetot C, Tertoolen LG and den Hertog / (2002) Rey .lon of receptor protein-tyrosine phosphatase alpha by o tive stre s. EMBO J. 21: 493-503

30. Casagrande S, Bonetto V, Fratelli M, Gianaž E, Eberini I, Massignan T, Salmona M, Chang G, I Imgren A and Ghezzi P (2002) Glutathionylation of human thiored ixin: issible crosstalk between the glutathione and thioredoxin syste Prou.nall. Acad. Sci. USA 99: 9745-9749

31. Fratelli M, Demol H, Puyp s asagrande ,Eberini I, Salmona M, Bonetto V, Mengozzi M, Duffieux F, Micles achi A, Vandekerckhove J, Gianazza E and Ghezzi P (2002) Ide cercation by h proteomics of glutathionylated proteins in oxidatively str ssed iman T lymphocytes. Proc. Natl. Acad. Sci. USA 99: 3505-3510

32. Beutler E (1991) G. se-6-phosphate dehydrogenase deficiency. N. Engl. J. Med. $3 \div$ - $169-174$

33. Luzzatto L. 751 Inherited haemolytic states: glucose-6-phosphate dehydrogen 2 se divíciency. Clin. Haematol. 4: 83-108

34. Robertson EJ (1987) Embryo-derived stem cell lines. In Teratocarcinomes and bryonic Stem Cells: A Practical Approach, Robertson EJ (ed) (Oxford, UK: II Press) pp. 71-112

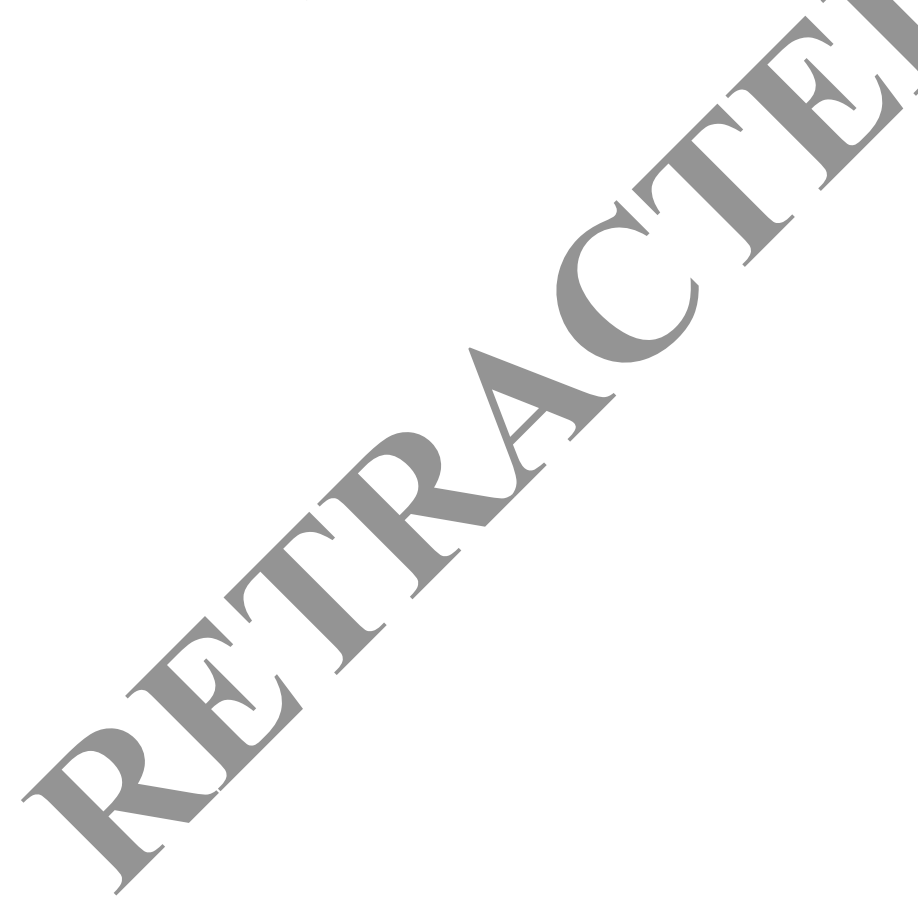

